\title{
The use of Petersen diagrams and period ratios in investigating the pulsational content of stars in the classical Instability Strip
}

\author{
E. Poretti and M. Beltrame
}

INAF - Osservatorio Astronomico di Brera, Via E. Bianchi, 46 - 23807 Merate (LC), Italy

\begin{abstract}
The aspects of the analysis of photometric time series obtained on double-mode or multiperiodic pulsating stars are briefly reviewed. In particular, the ratios between frequencies are used to pin cases revealing peculiarities. In addition to the Petersen diagrams, we also demonstrated that the period ratios can single out interesting objects. In particular, new results are obtained on High-Amplitude Delta Scuti stars contained in the OGLE-II database.
\end{abstract}

\section{Introduction}

In the recent years a huge collection of time series has been obtained on variable stars, as a noticeable by-product of several microlensing projects. Therefore, the investigation of thousands of light curves is carried out by detecting the frequencies present in the time series. It is not easy to give astrophysical depth to this kind of analysis, as we have monochromatic photometric data only at our disposal. Different classes of variable stars can show very different physical processes with very similar light curves. Moreover, instrumental terms are often superimposed to physical ones in the same frequency range. In this paper we will try to review some of these aspects.

\section{The Petersen diagrams}

When multiperiodicity is detected, it is quite an obvious procedure to calculate the frequency ratios. In case of double-mode pulsation, we deal with a short $\left(P_{S}\right)$ and a long $\left(P_{L}\right)$ period. The plot of the $P_{S} / P_{L}$ ratio versus $P_{L}$ is now known as the Petersen diagram (Petersen 1973). Small variations of temperature, mass, metallicity or luminosity push the $P_{L}, P_{S} / P_{L}$ points into different directions: as an example, see Fig. 2 in Popielski et al. (2001). The mass capable to reproduce the observed $P_{S} / P_{L}$ ratio is called the beat mass.

There have been several astrophysical applications of the Petersen diagrams. The most relevant one has been the reconciliation of pulsational, evolutionary and beat masses of Double Mode Cepheids after having introduced the new opacities (Christensen-Dalsgaard \& Petersen $1995)$. Let us indicate the fundamental radial mode as $F$, the first overtone radial mode as 10 , the second one as 20 .

Recently, Beltrame \& Poretti (2002) demonstrated that HD 304373 is the second case of $10 / 20$ double-mode Cepheid in the Galaxy. Its position in the Petersen diagram is very similar to that of some LMC stars. In general, all the $20 / 10$ values are very close to each other and only the different range of periods allows us to separate the different environments (Fig. 1, left panel). On the contrary, the $10 / \mathrm{F}$ ratios show a strong dependence on metallicity 
(Fig. 1, right panel). It seems that the $10 / 20$ ratios are less sensitive than the $10 / \mathrm{F}$ ratios to the difference in metallicity: the latter values comprise a five time larger interval than the former ones.

Christensen-Dalsgaard \& Petersen (1995) pointed out that the match between the F/1O ratios for galactic pulsators and the theoretical models occurs for metallicities smaller than the solar value of $0.017-0.020$; if that applies for the $10 / 20$ pulsators too, a metallicity close to 0.010 allows the $P_{2} / P_{1}=0.8058$ and $P_{1}=0.922405 \mathrm{~d}$ values to reasonably fit the theoretical models (Christensen-Dalsgaard \& Petersen 1995). We also note that assuming $P_{1} / P_{0}=0.70$, we obtain $P_{0}=1.32 \mathrm{~d}$, i.e., the fundamental period of HD 304373 is really one of the shortest among galactic Cepheids.
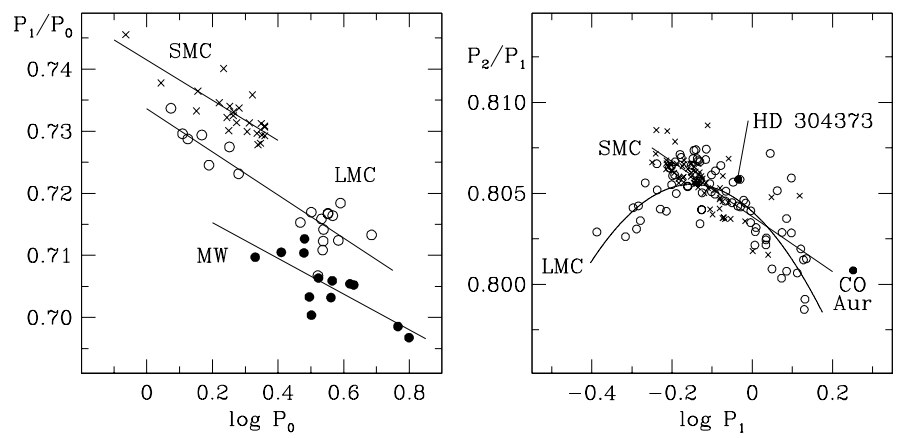

Figure 1: The Petersen diagrams for double-mode Cepheids in the Milky Way, the Small and Large Magellanic Clouds. The $P_{0} / P_{1}$ pulsators belonging to the three different galaxies are well separated, while the $P_{2} / P_{1}$ ones are not.

As in the case of CO Aur, there is no significant contribution of the $2 f_{2}$, harmonic in the light curve of HD 304373, i.e., it is perfectly sine-shaped within the error bars. Only small or marginal deviations from a sine wave have been found in the LMC and SMC 20 samples.

\section{The distinction between pulsating stars and geometric variables}

The analysis of the 82 periodic pulsating stars included in the OGLE II database (Mizerski \& Beijger 2002) revealed that pulsating and geometric variables can be confused by some automatic routines, but they can be more easily separated by more clever methods (Beltrame 2002). As a first step, we decided to perform frequency analyses of all the stars, to avoid any possible misinterpretation. Using the least-squares iterative sine wave fitting (Vaniček 1971), we obtained the power spectrum for every variable star of our sample, thus being able to compare the highest peaks with their aliases. No discrepancy was found as respects the values reported by Mizerski \& Beijger (2002).

The following step was to refine the periods of all the stars and to analyze their residual power spectrum, looking for the presence of multiperiodicity or other peculiar effects (such as the Blazhko effect for RRab and RRc). Therefore, we considered the preliminary solutions and the fit order found in the previous step. The solution was given as input parameters to a code keeping locked the relationships between the main frequency and the harmonic terms (Multiple Trigonometric Analysis Program; Carpino et al. 1987); the best fit was searched for 
around the preliminary solution. Once the refined period was obtained, we started searching for additional terms. Only the frequency values of the main term and its harmonics were considered as input values; no prewhitening was done. A few multiperiodic stars (RRab and RRc showing Blazhko stars, a new multimode pulsator) have been discovered. Moreover, the results of the period refinement shows that we have $\Delta P \sim 10^{-7}$ for 14 stars, $\Delta P \sim 10^{-6}$ for 43 stars. The rest of the sample (24 stars) showed a more significant refinement, with $\Delta P \sim 10^{-5}-10^{-4}$.
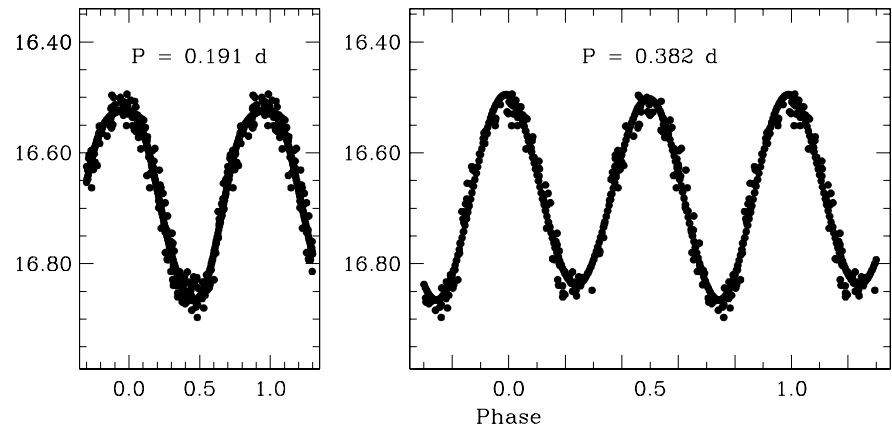

Figure 2: The true nature of bul1.686: the star is not a pulsating variable with a period of $0.191 \mathrm{~d}$ (left panel), but a geometric one with a period twice the "pulsational" one (right panel): note the different depths of minima.

Having obtained the correct periods for every star, we started with a preliminary fit up to the eighth order, in order to obtain the correct Fourier parameters. Therefore, we discarded all harmonics with amplitudes less than 3 times their error estimate. During this procedure, three stars (bul1.2457, bul1.1323, bul1.686), classified as HADS by Mizerski \& Beijger (2002), showed a notable spread of points at the minimum light of their light curves. Therefore, we checked the possibility for them being W UMa variables, doubling the period found. We found that bul1.686 is clearly a W UMa variable, since the light curve on the doubled period shows two minima having different depths (Fig. 2). In the other cases, there is still some dispersion at minima and a certain classification cannot be made. However, the power spectra always detected a pair of terms, in the ratio $2: 1$, with the higher amplitude related to the shorter period. A conservative approach would strongly suggest that in presence of the 2:1 ratio the variable should be considered a geometric one (eclipsing or rotational). Indeed, to explain such a ratio by the excitation of resonant modes seems an "ad hoc" solution, even if very attractive for theoreticians. We also note that stars showing a sine-shaped light curve (i.e., no significant contribution from the harmonics of the main frequency) can be geometric variables having minima of equal amplitudes. In case of monoperiodicity, this fact makes it quite delicate to distinguish the sine-shaped light curves of 20 pulsators (see previous section) from those of geometric variables.

\section{The frequency ratios in High Amplitude Delta Sct stars}

Poretti (2003) recently pointed out that HADS can show frequency spectra much more complicated than the ones considered some years ago. Figure 3 (which is an update of Fig. 5 
by Poretti 2003) illustrates how several stars deviate from the canonical 0.77 ratio between the $F-$ and the 10 modes. Values higher than 0.77 can be caused by very low metallicities $(Z<0.0005)$. Since most cases are actually much higher than 0.77 , the metal shortage should be very relevant and this seems unlikely for Pop. I stars. Also fast rotation can strongly modify the ratio, but it is quite uncommon in HADS stars, which are evolved ones. If we also consider the variety of ratios visible in Fig. 3, the excitation of nonradial modes is indicated as the natural explanation for such a different pulsational content. To corroborate the excitation of nonradial modes in HADS, high-resolution spectroscopy is strongly recommended to detect the signature of line profile variations.

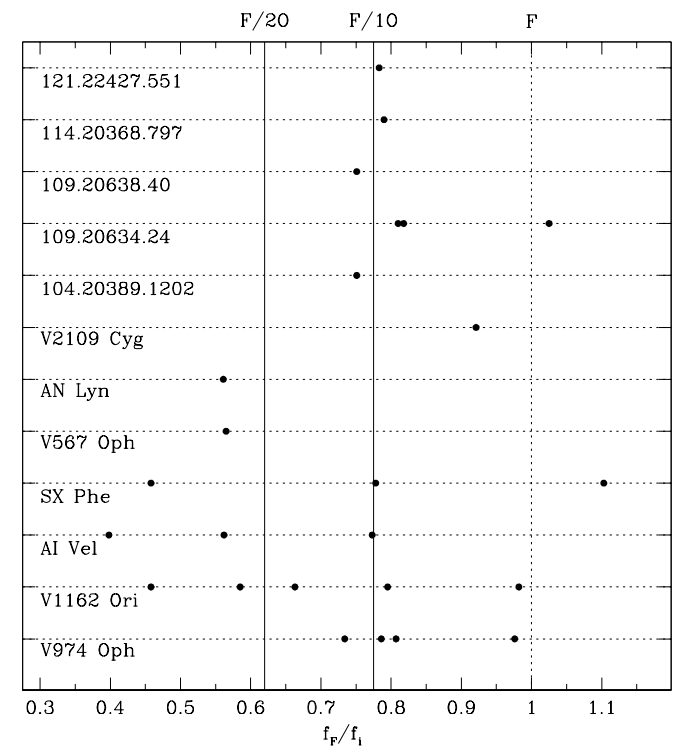

Figure 3: Observed (filled dots) frequency ratios among HADS stars showing possible nonradial modes. Vertical lines indicate the theoretical ratio between fundamental (F) and overtone (first, 1O; second, 2O) radial modes. Note also the ratios around 1.0, i.e., the presence of modes close to the fundamental radial one.

As discussed above, the 0.800 ratio is found in double mode Cepheids pulsating in the 10 and 20 modes. Such a value is also observed in some double-mode HADS stars (Musazzi et al. 1998) and it is attributed to the same modes, but there are some unclear points. In particular, the 0.800 ratio is related to an unusual shape of the $P_{L}$ light curve: the descending branch is steeper than the ascending one. This feature still remains unique in the zoo of the light curves of pulsating variables. The large databases did not supply any new stars simultaneously showing the 0.800 ratio and the asymmetric-in-the-bad-sense light curve.

When looking at frequency ratios it is interesting to search for the 0.620 value, which should be the signature of the excitation of the $F$ and 20 modes. Performing the search 


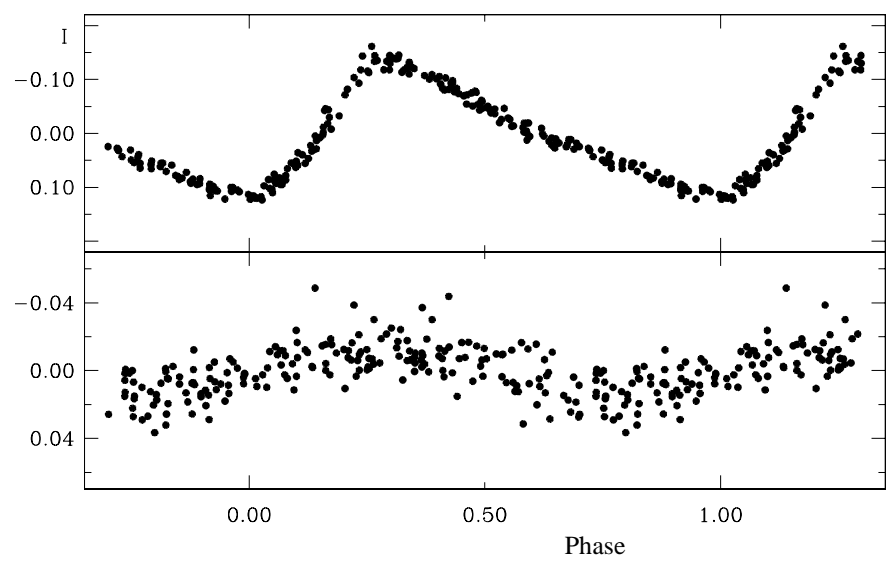

Figure 4: The phased light curves of two frequencies detected in the bul1.3074 time series: they give $f_{1} / f_{2}=0.62$

described above in the OGLE-II database, we discovered that bul1.3074 is a new multiperiodic HADS star. Three independent frequencies have been detected, i.e., $f_{1}=5.275, f_{2}=8.672$ and $f_{3}=8.618 \mathrm{~cd}^{-1}$. In addition to the close doublet composed of $f_{2}$ and $f_{3}$, the ratio $f_{1} / f_{2}$ is intriguing, as it results in 0.61 . The presence of aliases could have interfered in determining the correct frequencies, but even considering the peaks at $f+1, f-1 \mathrm{~cd}^{-1}$ we were not able to solve the problem detecting the usual 0.77 ratio. Also in this case a possible explanation could be the excitation of a nonradial mode of pulsation. However, if $\mathrm{F} / 2 \mathrm{O}$ pulsators do exist among HADS, bul1.3074 should be considered a promising candidate. Figure 4 shows the light curves of the $f_{1}$ and $f_{2}$ terms: they look very well defined, strongly supporting their reality.

Acknowledgments. This paper was partly prepared during EP's stay at Konkoly Observatory in the framework of the Italian-Hungarian TéT cooperation (project I-24/1999).

\section{References}

Beltrame, M., 2002, Laurea Thesis, Università di Milano (in Italian)

Beltrame, M., Poretti, E., 2002, A\&A 286, L9

Carpino, M., Milani, A., Nobili, A.M. 1987, A\&A 181, 182

Christensen-Dalsgaard, J., Petersen, J.O., 1995, A\&A 229, L17

Mizerski, T., Beijger, M., 2002, Acta Astron. 52, 61

Musazzi, F., Poretti, E., Covino, S., Arellano Ferro, A., 1998, PASP 110, 1156

Petersen, J.O., 1973, A\&A 27, 89

Popielski, B. L., Dziembowski, W. A., Cassisi, S., 2000, Acta Astron. 50, 491

Poretti, E., 2003, A\&A 409, 1031

Vaniček, P. 1971, Ap\&SS 12, 10 